\title{
Change Detection and Extraction of Information in Remote Sensing Images Using Time Series Information
}

\author{
Aarti Karandikar ${ }^{*}$, Abhishek Sambhare², Niladri Sen ${ }^{3}$, Aditya \\ Kakde $^{4}$, Saket Gulhane ${ }^{5}$, and Saurabh Thakre ${ }^{6}$ \\ ${ }^{* 1,2,3,4,5,6}$ Shri Ramdeobaba College of Engineering and Management Nagpur India
}

\section{ABSTRACT}

Over the years, change detection(CD) has been garnering a lot of attention due to its applications in remote sensing to detect changes in multitemporal satellite imagery. High-resolution images are taken using satellites which provides multispectral as well as hyperspectral image data. Using change detection techniques, various changes occurring over time in these images in different scenarios can be detected, which in turn, help to track changes in vegetation cover, geology, urban development, etc. Various CD techniques have been discovered over the years. In this study, the authors present a novel idea to use Principal Component Analysis (PCA) and k-means clustering to detect and visualize changes occurring over a certain time period using multitemporal satellite images. Our methodology depicts how a satellite image with a really high resolution can be used in change detection by first reducing the dimensions using the PCA algorithm and then further using its features to segregate the points into various classes.

KEY WORDS: CHANGE DETECTION, PRINCIPAL COMPONENT ANALYSIS, REMOTE SENSING, MULTITEMPORAL AND BINARYMAP.

\section{INTRODUCTION}

One of the interesting application of image processing is to detect changes in images acquired over a period of time of a particular region. To detect changes in surface features of a region, the algorithm is feed with multiple images of the same target. These images can be singlephase or multi-band remote sensing images. Satellite imagery is prominently used to monitor urban expansion and land use land cover changes at a medium or large scale. This aids in better observation and understanding of the evolution of urbanization and helps in advancing the sustainable development process. The main objective

\section{ARTICLE INFORMATION}

Received 15th Oct 2020 Accepted after revision 27th Dec 2020 Print ISSN: 0974-6455 Online ISSN: 2321-4007 CODEN: BBRCBA

Thomson Reuters ISI Web of Science Clarivate Analytics USA and Crossref Indexed Journal

\section{Clarivate
Analytics}

NAAS Journal Score 2020 (4.31) SJIF: 2020 (7.728)

A Society of Science and Nature Publication,

Bhopal India 2020. All rights reserved.

Online Contents Available at: http//www.bbrc.in/

Doi: $h$ ttp://dx.doi.org/10.21786/bbrc/13.14/18 of change detection is to identify the changes in the spatial representation of any point by observing it at different times.

Typically used change detection methods are either to utilize post-classification analysis or use difference image analysis. The post-classification method first classifies the contents of two temporally different images of the same scene and then compares them to identify the differences. One may get inaccurate results due to classification errors in either of the two images. To combat this, a high degree of accuracy is required of the classification. This study is using the concept of image segmentation. It basically divides a given input image into segments which simplifies the image analysis process. The segments comprise of sets of pixels or "super-pixels" and represent objects or parts of objects.

Segmentation simplifies / changes the representation of an image into something that is more meaningful and easier to analyze. Image segmentation is normally used to locate objects and boundaries, i.elines, curves, etc.,

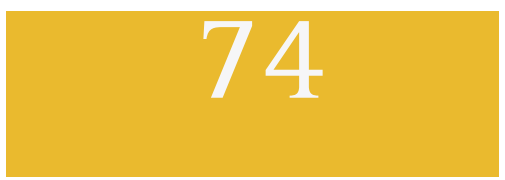


in images. More specifically, image segmentation is the process of assigning a label to every pixel in an image such that pixels with the same label share certain distinct characteristics. It groups pixels into larger components and thus eliminate the need to consider individual pixel as units of observation. The two types of image segmentation are Semantic Segmentation and Image Segmentation. In this paper semantic segmentation is used. Semantic Segmentation is performed on remote sensing images using U-Net architecture. It is based upon semantic segmentation. It is symmetric and skips connection between upsamplingpath and downsamplingpathand applies concentrator operator instead of sum operator. It is divided into 3 parts - downsampling path, bottleneck, and the upsampling path.

\section{MATERIAL AND METHODS}

The workflow is distributed across 2 phases: in the first phase all the changes are detected and a binary map is created to better visualize the changes; and in the second phase highly trained models are used to classify the changes into different types of objects/classes. Using these features, it can be detected what places, objects, geometries, etc are either added or removed. The dataset was downloaded from Amazon Web Services (AWS) Public Dataset which is publicly available containing commercial satellite imagery. The satellite imagery is provided by Digital Globe, a vendor of space imagery and geospatial content. Raster data in the SpaceNet dataset is present in the form of .tif (GeoTiff) images. These GeoTiff images are made using multispectral image data. SpaceNet dataset provides:

- MS: Raw source GeoTIFF of 8 Band Multispectral raster data from WorldView-3.

- PS-MS: Raw source GeoTIFF and COGs of 8 Band Multispectral raster data pan-sharpened to $0.3 \mathrm{~m}$.

- PAN: Raw source GeoTIFF of Panchromatic raster data from Worldview-3.

- PS-RGB: Raw source GeoTIFF of RGB raster data from Worldview-3 pan-sharpened to $0.3 \mathrm{~m}$.

- Metadata (GeoJson files).

- The geotiff images for Vegas, Paris, Khartoum, etc locations were selected to experiment with.

Phase 1: In this Phase PCA algorithm is implemented for change detection on multitemporal remote sensing images. We have implemented principal component analysis (PCA) for dimension reduction. PCA takes less time to implement and provides better accuracy. Block structure is used of the correlation matrix so that the PCA is conducted on data of smaller dimensionality, which significantly reduces the computational load and gives a smaller set. This consists of features which are uncorrelated to each other. The multispectral images consists of multiple bands are are acquired in different parts of the electromagnetic spectrum. Using PCA data can be transformed into smaller sets, $\mathrm{N}$ number of correlated bands of the image data can be reduced to a few uncorrelated bands.
Following steps are performed to obtain the change map for two images:

Difference image and the Eigenvector space (EVS): The difference of the two images gives an image wherethe pixels linked with changes would have values that are considerably different from those of the pixels associated with unchanged areas. Non overlapping blocks are taken from the obtained difference image and are flattened into row vectors. To get the Eigenvector space(EVS), principal component algo is then applied on this vector set. Building the Feature Vector Space (FVS): We first create VS by taking blocks from the difference image, flatten them, only this time, the blocks are overlapping. FVS is then constructed by projecting VS onto the EVS which can be done by the following matrix multiplication:

\section{$\mathrm{FVS}=\mathrm{VS} . \mathrm{EVS}$}

Clustering the FVS: The feature vector carries data about changed and unchanged pixels, which are clustered into two classes using K Means clustering. The cluster which belongs to the changed class is the one with the lowest number of pixels, and the highest mean. Using these, a change map is created representing the changes.

$$
\text { change_map }(i, j)=\left\{\begin{aligned}
255, & \text { if }(i, j) \in \text { pix }_{u} \\
0, & \text { otherwise }
\end{aligned}\right.
$$

Phase 2 - Feature Extraction: In this phase features from the remote sensing images were extracted to classify the change in different objects/classes. For the Extraction of features, Semantic Image Segmentation is done using U-NetArchitecture. The architecture of a U-net is similar to the encoder-decoder architecture, with additions of skip connections. The first half focuses on feature extraction(analysis) and the next half is used for localization and segmentation(synthesis).

Figure 1: U-Net Architecture [7]

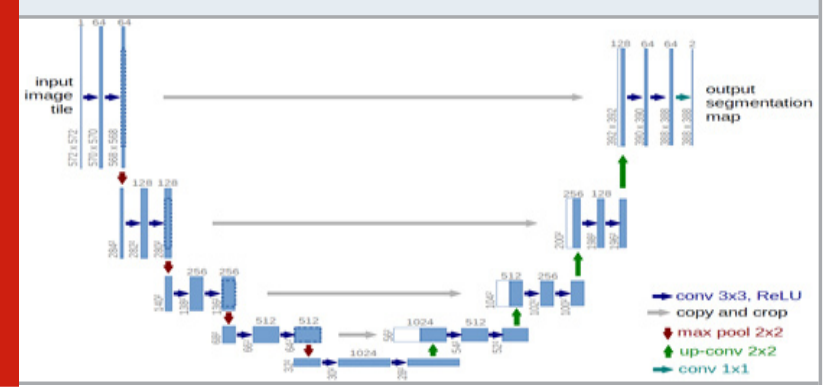

As discussed, in order to better localize and learn representations, whileperformingupsampling and going deeper in the network, the higher resolution features are concatenated from downpart with the upsampled features, with following convolutions. Since upsampling is a sparse operation it needs a good priorknowlwdge from earlier stages for better representing the localization. Unlike in CNNs, where it only learns the feature mapping of images by converting them into vectors, here it is also needed to reconstruct an image from this vector 
in segmentation. This is a very arduous task. So, while converting an image into a vector, the feature mappings of the image that were already learned in the first half are used to convert it again to an image. This is the idea behind U-Net. Use the same feature maps that are used for the contraction to expand a vector to a segmented image.

Figure 2: Sample satellite images from two different timestamps.

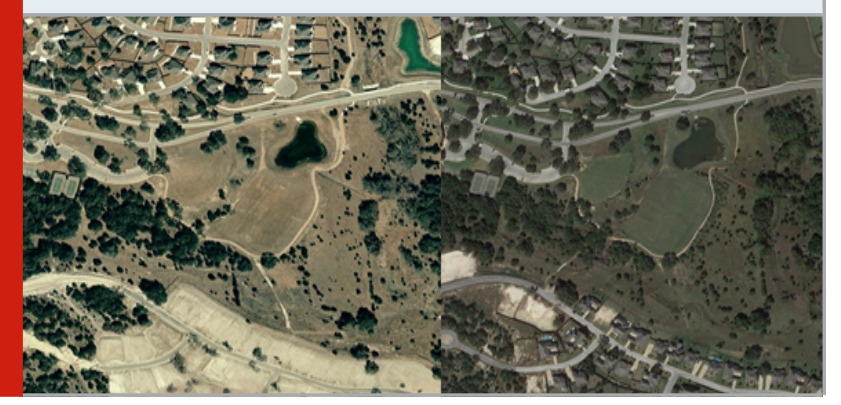

Figure 3: Change Map of above two satellite images

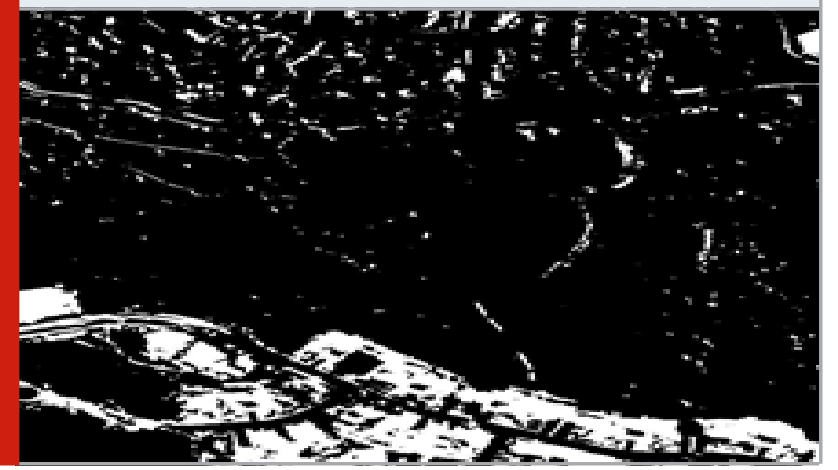

Figure 4: Change Map with sharpened edges

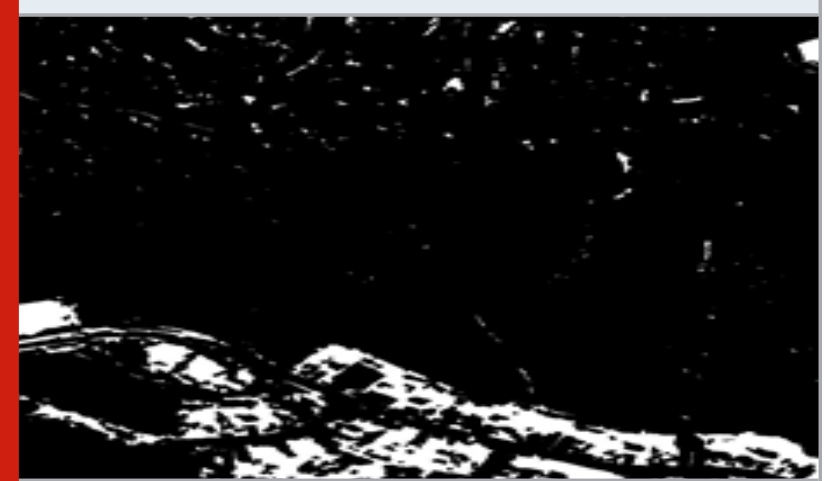

RESULTS AND DISCUSSION

Change detection was performed on the multitemporal remote sensing images. The Principal Component Analysis (PCA) calculation was applied to two sample satellite imagery of two different timestamps. As shown in Fig. 2 there are two sample satellite images of different timestamps on which change detection was carried out. The image shown in Fig. 3 was obtained from these two images, which shows the changed portion or pixels in white color and unchanged in black color. The image shown in Fig. 4 is cleaned by sharpening the edges to get a better view of change.

As shown in Fig 2. It provides the change map which detected the change in two images of Dubai of different timestamps. The change is shown in white color. For the feature extraction the model was trained using the spacenet dataset. The model is capable of detecting some objects/classes. It classifies or extracts the information using semantic segmentation which was carried out using U-Net Architecture. The input image of 640 x 640 $\mathrm{x} 3$ was passed to the model which then using U-Net architecture gives the output image of $640 \times 640 \times 1$ that is in binary map. As shown in figure 5 the output image obtained shows the building detected in white color.

Figure 5: Building classified from the remote sensing image

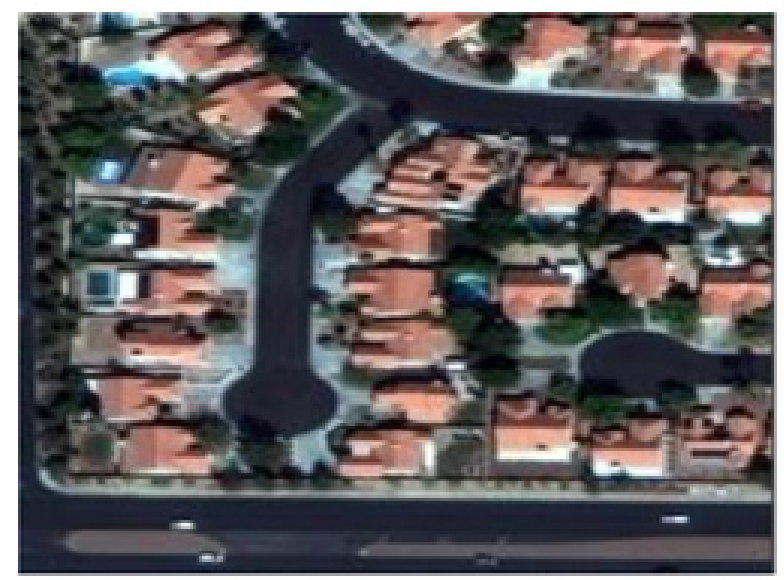

Input: $640 \times 640 \times 3$

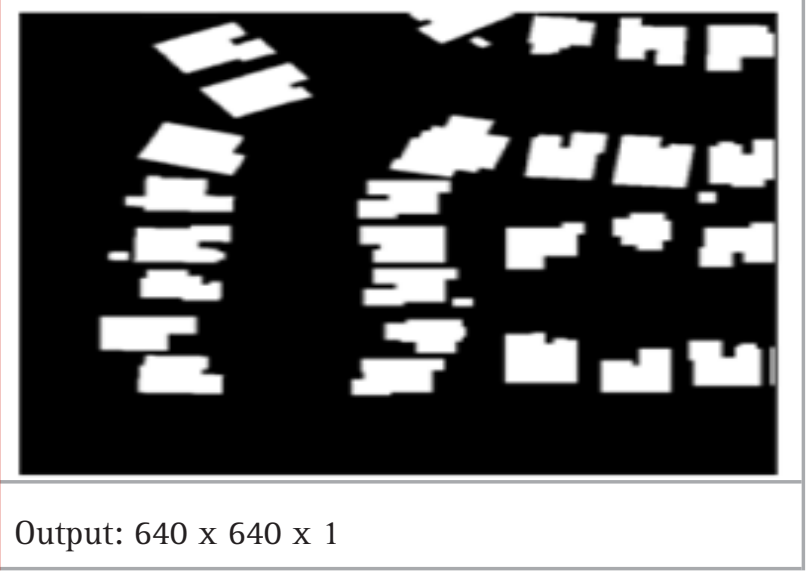

\section{CONCLUSION}

In this project changes are detected in remote sensing images of satellites using time series data. This project was done in 2 phases. First, was the change detection part and the second phase was all about feature extraction. In the first phase, change detection was done using the 
Principal Component Analysis (PCA) Algorithm. PCA was efficient in terms of accuracy, as it provides better accuracy in remotely sensed image classification. The PCA based system provides high speed processing and comparatively better accuracy. PCA could also handle large numbers of image data due to its capability of reducing data dimensionality and complexity. In the second phase we extracted the features from satellite images using Semantic segmentation and U-Net architecture. Semantic segmentation identifies what is present and where, it also clusters the patches of images which have the same object of interest. The U-Net architecture is used with Semantic segmentation, it follows an autoencoder architecture as it has 2 structures. The U-Net joins the area data from the downsampling way with the relevant data in the upsampling way to at last acquire an overall data consolidating localisation and setting, which is important to foresee a decent division map. Hence, it provides the information of features extracted from images to classify the change in objects or classes in remote sensing images.

\section{REFERENCES}

B. K Tripathy, Sudhir Sahu, MBNV Prasad. PCA Classification Technique of Remote Sensing Analysis of Colour Composite Image of Chilika Lagoon, Odisha. International Journal of Advanced Research in Computer Science and Software Engineering. Vol 5 Issue 5, 2015, pages 513 - 518 .

CelikTurgay. Unsupervised Change detection in Satellite images using Principal Component Analysis and K-means clustering. IEEE Geoscience and Remote Sensing Letters, Vol. 6, 2009.
D.RPanuju, D.JPaull,A.LGriffin. Change Detection Techniques Based on Multispectral Images for Investigating Land Cover Dynamics. Remote Sens. 2020, Vol12, 1781; doi:10.3390/rs12111781.

Javier Estornell, Jesus M. Mart '1-Gavil 'a, M. Teresa Sebasti 'a, Jesus Mengual. Principal Component Analysis Applied to Remote Sensing. Modelling in Science Education and LearningVolumen 6(2), No. 7, 2013, pages 83 - 89.

K.Vaswani and A. Karandikar. An Algorithm for Spatial Data Mining Using Clustering. Journal of Engineering and Applied Sciences, Vol 12(Special Issue 12), 2017. Pages 9572-9575.

MKDolati and AEslamBonyad. Use of Principal Component Analysis in Accuracy of Classification Maps (Case Study: North of Iran). Research Journal of Forestry, Vol10, 2016 pages 23-29.

Mishra S, Shrivastava P, Dhurvey P. Change Detection Techniques in Remote Sensing : A Review. International Journal of Wireless and Mobile Communication for Industrial Systems Vol. 4, No. 1 (2017), pp. 1-8

Olaf Ronneberger, Philipp Fischer, and Thomas Brox. U-Net: Convolutional Networks for Biomedical Image Segmentation. Medical Image Computing and Computer Assisted Intervention, Springer, LNCS, Vol.9351, 2015, pages 234-241.

Sony Ahuja and A. Karandikar. Improving the Efficiency of Forest Cover Forecasting using ANFIS. International Journal of Innovative Technology and Exploring Engineering , Volume-8 Issue-8S3, 2019, pages 282287. 